\title{
Cardio-Vascular Consequences of Parathyroid Disorders in Adults
}

Jens Bollerslev ${ }^{1,2}$, Evelina Sjöstedt ${ }^{3,4}$, and Lars Rejnmark ${ }^{5,6}$

${ }^{1}$ Section of Specialized Endocrinology, Division of Medicine, Oslo University Hospital and

${ }^{2}$ Faculty of Medicine, University in Oslo, Oslo, Norway; ${ }^{3}$ The Department of Immunology, Genetics and Pathology, Uppsala University, Uppsala, Sweden; and ${ }^{4}$ Department of Neuroscience, Karolinska Institute, Stockholm, Sweden ${ }^{5}$ Department of Clinical Medicine, Aarhus University and ${ }^{6}$ Department of Endocrinology and Internal Medicine, Aarhus University Hospital, Aarhus, Denmark.

Correspondence:

Jens Bollerslev, MD; DMSc

Professor in Endocrinology

Faculty of Medicine

University in Oslo

Box 1072 Blindern

0316 Oslo, Norway

jens.bollerslev@medisin.uio.no 


\begin{abstract}
PTH is a metabolic active hormone primarily regulating calcium and phosphate homeostasis in a very tight and short term-manner. Parathyroid disorders in adult patients reflect a variety of different conditions related either to the parathyroid glands itself or to the effects of the secreted hormone. The clinical specter varies from the common disease primary hyperparathyroidism (PHPT) to the orphan conditions Pseudohypoparathyroidism (Ps-HypoPT) and chronic Hypoparathyroidism (HypoPT). The purpose of this review is to describe the consequences of disturbances in levels or action of PTH for cardiac function and cardiovascular risk in adult patients with these disorders.
\end{abstract}

Most patients with PHPT achieve the diagnose by chance and have minor or no specific symptoms. Still, these patients with mild PHPT do possess cardiovascular (CV) morbidity, however so far not proven ameliorated by surgery in controlled trials. In severe cases, the CV risk is increased and with a potential reversibility by treatment.

Patients with Ps-HypoPT have resistance to PTH action, but not necessarily total resistance in all tissues. So far, no clear CV morbidity or risk has been demonstrated, but there are several aspects of interest for further studies.

Most patients with HypoPT do get their hormonal deficiency syndrome following neck surgery. These patients do experience multiple symptoms and do have an increased CV-risk before the primary surgery. Based on existing data, their CV mortality do not deviate from the expected when adjusting for the preexisting increased risk. Patients with nonsurgical (NS-) HypoPT do demonstrate increased CV-risk also associated with exposure time.

Endocrine disorders with alterations in PTH function have major impact on the cardiovascular system of importance for morbidity and mortality, wherefore management of these specific diseases should be optimized currently, as new data become available, however also avoiding over-treating asymptomatic patients. 


\section{Introduction}

Parathyroid disorders in adult patients reflect a variety of different conditions related either to the parathyroid glands itself or to the effects of the secreted hormone, PTH (1). PTH is the major hormonal regulator of the tightly bound calcium homeostasis via a direct effect on the kidney and bone calcium fluxes, and indirectly via activation of vitamin D enhanced calcium absorption from the gut (2-5). PTH acts mainly by binding to the specific PTH/PTHrP binding receptor PTHR1 (6), a common G-protein coupled receptor. According to the Human Protein Atlas (http://www.proteinatlas.org/), (7) PTHR1 is mainly and abundantly expressed in the kidney, and at much lower levels in several other tissues such as liver, adrenal, and potential also at very low levels in the cardiomyocytes. The protein is located in the renal tubules, shown by unpublished Immunohistochemical images (antibody HPA075879, Atlas antibodies, complete protein profile will be published in the next update of the Human Protein Atlas), see Figure 1a. Although RNA expression is detected at low levels in several tissues, the protein can only be detected in kidney. PTHR2 is also a G-protein coupled receptor, specifically binding PTH as the ligand. The tissue distribution is less general than for PTHR1, PTHR2 RNA expression is mainly detected in the brain, bone marrow and salivary glands according to the Human Protein Atlas (http://www.proteinatlas.org/), or mainly detected in brain, placenta, pancreas and testis according to Usdin et al (8). Antibody-based protein localization (antibody HPA010655, Atlas antibodies) detects PTHR2 abundantly in the pancreas as well as endothelial cells throughout the body (Figure 1b), although the protein location needs to be further validated. Neither of the receptors seems to be expressed in higher amounts in the heart and only PTHR2 show positivity in the vasculature.

Being the principal regulator of calcium homeostasis, PTH and calcium levels go hand in hand as risk factors for various outcomes, including cardio-vascular (CV) morbidity and mortality. Early epidemiological studies identified high calcium levels as a risk factor for overall mortality in the general population, and especially as a risk for CV-mortality in men (9). In accordance, calcium level has been shown to be an independent risk factor for myocardial infarction in middle aged men (10), also confirmed in the Troms $\varnothing$ study (11). These studies do not show causality and are hypotheses generating only. But it is of interest that PTH levels seem to reflect overall CV-risk in the general male population, even within the normal range (12). Accordingly, in a large clinical epidemiological study of patients of both sexes undergoing coronary angiography (a third of the patients did not have coronary disease) PTH was shown to be an independent predictor of mortality and cardiovascular events. A special strong association was 
found between PTH and sudden death (13). These data points towards a more general metabolic effect of PTH than merely controlling calcium homeostasis, and a positive relation between PTH and leptin has been suggested, at least in primary hyperparathyroidism (PHPT) (14).

The clinical spectrum of parathyroid disorders potentially affecting the heart and cardiovascular system in adults spans from the very rare syndromic disorders related to resistance to PTH action as in pseudohypoparathyroidism (Ps-HypoPTP) (15), to the highly prevalent disease PHPT, with a prospectively proven prevalence between 2 and $5 \%$ in peri-and postmenopausal Scandinavian women $(16,17)$. Recently, renewed scientific attention has been payed to chronic hypoparathyroidism in adults (HypoPT) (18), due to an ongoing discussion on management. The purpose of this paper is to review the consequences of disturbances in levels or action of PTH for cardiac function and CV-risk in adult patients with these well-defined clinical entities. We will focus on areas of special clinical interest where recent advances have been gained with potential implications for clinical management.

\section{Primary Hyperparathyroidism (PHPT)}

In PHPT, calcium levels as well as PTH levels are inappropriately increased in relation to each other in a stable situation with only little daily variation in calcium level, a condition termed equilibrium hypercalcemia (5). Thus, in patients observed for years without treatment for PTHT, calcium levels are remarkable stable underscoring the concept of a set-point error in PHPT (5, 19-21).

\section{PTH or Calcium and $C V$-risk}

Well knowing that PTH have wide metabolic effects, directly or indirectly and that calcium levels are correlated to vascular calcification and CV risk, the question remains whether it is PTH or calcium by itself that is the important player; most probably a combination (22). To illustrate this point, in a large case-control study of PHPT from the epidemiological wellcharacterized and representative area Tayside in Scotland, $Y u$ and coworkers followed 2.300 patients for a total of more than 7.300 observation years, of whom about $90 \%$ were followed without surgery (23). The study found that PTH level at baseline - not calcium levels, was an independent predictor of all-cause mortality, fatal and non-fatal CV-disease (CVD). These hypotheses generating data are in accordance with other studies showing a correlation between PTH and arterial stiffness (24) and left ventricular mass (LVM) (25) in the so called mild form of PHPT, where the diagnosis often is made by chance in otherwise asymptomatic patients (26). Epidemiological studies from Denmark have shown a clear increased CV-risk in PHPT patients 
with higher calcium levels (27), also indicating a potential threshold level, below which the risk was not increased.

Management of mild PHPT with only borderline increased calcium levels has been a matter of discussion for decades and several international consensus conferences have given indications for surgical treatment or observation without intervention (28). So far, the recommendations have focused on total calcium levels only, not considering PTH levels, nor the fact that total calcium might underestimate free calcium levels in PTHP (29).

The risk of CVD is increased in non-mild PHPT, as evidenced in several studies (30-32), but not confirmed in others $(33,34)$, the latter pointing at the potential threshold level (34). LVM by Echocardiography is a strong predictor of CVD and CV-mortality (35), and LVM has been found increased in most studies of non-mild PHPT $(36,37)$, but not in the nowadays most prevalent mild form of PHPT (38). In the Scandinavian Study on Primary Hyperparathyroidism (SIPH), a randomized controlled study of observation versus surgery in mild PHPT, we found a strong correlation between PTH a baseline (not calcium) and LVM and an indication of a treatment effect of surgery on ventricular dimensions two years after successful surgery (25). A recent meta-analysis on the effects of parathyroidectomy (PTX) on LVM in patients with PHPT found a significant reduction in cardiac dimensions by surgery, and the improvements were correlated with PTH levels, so the higher preoperative PTH, the better response (39). In other words, PTH seems to have a trophic effect on heart dimensions in PHPT, partly reversible by PTX, potentially above certain, yet so far not well-defined threshold.

\section{Arterial Hypertension}

Arterial hypertension is prevalent in PHPT, related to as well PTH as calcium levels, whereas the exact mechanism remains elusive, as reviewed recently (40). Potential mechanisms related to the RAS-system or structural changes in the vasculare were discussed. Of interest, PHPT by itself predicts arterial hypertension in the general population (41), and a recent case-control study of hypertensive patients with or without PHPT demonstrated that patients with PHPT had an increased blood pressure (BP) morning raise compared to non-PHPT hypertension patients (42). The morning BP surge was independently associated with calcium levels and markers of the metabolic syndrome.

Traditionally, PHPT has been linked to the metabolic syndrome including arterial hypertension (43), and improvement in BP following PTX has been demonstrated in several studies on nonmild PHPT (Total calcium levels more than $0.25 \mathrm{mmol} / \mathrm{l}$ above the upper limit of normal or indications for surgery (28)), (44-46). However, in a randomized controlled study, EjlsmarkSvensson et al investigated the short-time effect (3 months) of PTX on BP in PTH patients with 
indications for surgery (47). In this controlled study, no beneficial effect on BP of surgery was found. Conversely, an increased in diastolic BP was found following surgical treatment based on 24 hour measurements (47). When it comes to mild PHPT, the few published randomized, controlled studies have not demonstrated an unequivocal treatment effect of surgery compared to observation without treatment on BP, with a follow-up of five years $(48,49)$.

In conclusion on arterial hypertension, patients with PHPT do have a higher risk of arterial hypertension related to several mechanisms of which the interplay with the RAS system might be of major importance (50). There seems to be an association between the PTH/calcium levels and the degree of hypertension (dose-response), and with a potential reversibility of the hypertension by surgery.

Reversibility of $C V$-risk in PHPT

The clinical manifestations of PHPT has arbitrarily been divided in three categories, normocalcemic (NC-), mild and classical/severe PHPT related to symptoms and calcium levels (28). Whether NC-PHPT is a preclinical form of PHPT that might or might not develop further is still discussed $(40,51,52)$. As the diagnosis of NC-PHPT relies on measurement of an elevated PTH concentration in spite of normal calcium level, and as PTH as other peptide hormones do fluctuate with time and sampling condition (53), the sensitivity of the diagnostic setup is essential (51). The sensitivity can be improved by adding a biochemical marker of bone turnover $(51,54)$, implying the impact of the hormone on target tissues. When it comes to CVrisk markers, NC-PHPT behaves like hypercalcemic PHPT at baseline and following surgical treatment (55). Thus, biochemical markers of CV-risk are increased in NC-PHPT and improves following treatment (55), as also seen in mild to moderate PHPT (47), but do not normalize (55). Arterial stiffness as measured by pulse wave velocity (PWV) is as mentioned increased in mild PHPT (24), with potential improvements following surgery depending on the degree of PHPT (47, 56-58).

Long-term treatment effect of surgery on CV-risk markers in the debated mild form of PHPT was recently published based on five-year data from the Scandinavian study (SIPH), (59). Out of the original 191 patients with mild PHPT randomized to either immediate surgery or observation without intervention, 119 concluded the five-year follow-up. A variety of CV-risk markers were analyzed longitudinally, including body composition by DXA and detailed cholesterol and glucose metabolism. Except for the expected increase in 25-hydroxy vitamin D levels following surgery, no significant treatment effect of surgery was demonstrated (59). The study is hampered by several caveats. However, it is still the largest and ever longest lasting randomized controlled study on mild PHPT. 
In conclusion, different markers of CV-risk are increased in PHPT, and presumably associated with markers of disease activity (calcium and PTH?). Following surgery improvements have been observed, but so far not clearly demonstrated to be normalized. Again, the literature points to a potential threshold effect, which however is not well documented.

\section{Pseudohypoparathyroidism (Ps-HypoPT)}

The underlying mechanisms of Ps-HypoPT are de novo or autosomal dominantly inherited inactivating genetic variants or epigenetic alterations in the GNAS locus (located on chromosome 20q13.32) causing resistance to peptide hormones acting through G proteincoupled receptors. The hallmark of the disease is target organ resistance to PTH causing (very) high PTH levels with hypocalcemia and hyperphosphatemia. The disease has an estimated prevalence of less than 1.1 per 100,000 (15).

Due to resistance to PTH, high PTH levels may not result in classical adverse effects of hyperparathyroidism. However, data suggest that not all tissues exhibit a full resistance to the hormone. Because of the rarity of the disease, only few studies are available on the sensitivity of the cardiovascular system to the high PTH levels, and whether patients with Ps-HypoPT are at increased risk for CVD. A case-series published back in the 1980's suggested a high risk of arterial hypertension defined as an office blood pressure above $155 / 90 \mathrm{mmHg}$, which was found in 53\% of the 46 patients with Ps-HypoPT (60). Furthermore, Ps-HypoPT has been associated with high/high-normal levels of cholesterol, LDL and triglycerides (61). However, findings from a more recent case-series including 30 Ps-HypoPT patients did not support such harmful effects of Ps-HypoPT (62). Only $20 \%$ of the patients were diagnosed with hypertension and only $10 \%$ were on treatment with cholesterol lowering drugs. Furthermore, office as well as $24 \mathrm{~h}$ ambulatory blood pressures and arterial stiffness (PWS) were similar to patients diagnosed with non-surgical hypoparathyroidism (i.e. patients with low PTH levels). Additionally, despite patients with Ps-HypoPT had significantly higher PTH levels compared to non-surgical hypoparathyroidism (19.8 \pm 31 vs. $2.1 \pm 1.6 \mathrm{pmol} / \mathrm{L}$, respectively), Ps-HypoPT was associated with a significantly lower pulse rate. Accordingly, the well-known chronotropic effects of PTH does not seem to affect patients with Ps-HypoPT (63).

Lack of detrimental CV effects of high PTH levels in Ps-HypoPT are further supported by findings from an epidemiological study taking advantages of the Danish National Hospital Patient Registry (64). The study compared all patients diagnosed with Ps-HypoPT in Denmark 
$(\mathrm{N}=60)$ with three times as many age- and gender matched population based controls. Included patients had hypocalcemia (mean $0.95 ; 95 \% \mathrm{CI}, 0.85$ to 1.05 [reference interval 1.18 to 1.32 ] $\mathrm{mmol} / \mathrm{L}$ ) with high PTH levels (mean 32.5; 95\% CI, 25.1 to 39.9 [reference interval 1.6 to 6.9] $\mathrm{pmol} / \mathrm{L}$ ). Mortality and risk of CVD including risk of ischemic heart diseases, arrhythmia and stroke did not differ between Ps-HypoPT patients and controls supporting that high PTH levels in Ps-HypoPT are not associated with harmful effect on the CV system.

\section{Chronic hypoparathyroidism in adults (HypoPT)}

HypoPT is per definition an orphan disease (as Ps-HypoPT) with an estimated prevalence about 30 per 100.000 inhabitants, most often secondary to neck surgery (65-67). Non-surgical (NSHypoPT) is caused by rare genetic disorders often diagnosed in childhood or adolescence, due to hypocalcemic symptoms (68). Clinically, and with respect to late CV complications surgical and NS-HypoPT reflect two different pathological conditions. Both conditions characterized by a two hormone deficient state - the lack of PTH and activated vitamin D, by low-to-low-normal calcium and by high phosphate levels, potential with an increased calcium-phosphate product (1). It is of interest that patients with postsurgical HypoPT seems to harbor more symptoms than NS-HypoPT, as given by studies on quality of life (65). These findings however, do not necessarily reflect morbidity in the heart and CV-system. Hypocalcemia by itself can lead to potentially reversible cardiomyopathy, in adults most often caused by HypoPT (69).

Management of patients with HypoPT has until recently been based on un-physiological treatment with mainly activated vitamin D and calcium supplement, in reality resulting in an overflow hypercalcuria $(18,70)$. Recently however, substitution with the lacking hormone PTH has been approved thereby potentially replacing both the lacking hormones, and hopefully improving long-term outcome of this debilitating condition $(1,18,70,71)$.

\section{Cardiovascular disease and risk}

Because of the rarity of HypoPT, data on CV morbidity and risk are scares and mostly based on register studies or retrospective case-control studies. Recent epidemiological studies on patients with NS-HypoPT from Denmark have shown an increased risk of heart disease, as ischemic heart disease and arrhythmia, but also stroke. Overall mortality was however, not increased. The study was based on 180 patients, of whom 123 were alive at the date of followup (mean age and mean follow-up time nearly 50 years). The sex distribution was almost equal, $47 \%$ being males (68). In another nationwide Danish follow-up study of patients with postsurgical HypoPT however, no increased risk for CV-disease was found (67), despite a 
clearly increased risk for renal complications and cataract. This study was based on patients developing postsurgical HypoPT following neck surgery for non-malignant disease, and identified totally 688 patients ( $88 \%$ females) with a mean age at diagnosis of 49 years. The patients were well matched by normal controls, with approximately the same follow-up time (8.4 vs 7.9 years). The cases had an increased risk of CVD before the surgery leading to HypoPT as compared to the controls. When adjusting for the more prevalent CVD prior to surgery in the Cox proportional-hazards models, the risk for CVD was not increased in the patient group. Neither in the crude model, was overall mortality not different between patients and controls (67).

These two studies points to the pathophysiological differences between postsurgical and NSHypoPT $(67,68)$, reflecting the potential devastating organ manifestations of the disease or its management. In NS-HypoPT with the longer disease duration, the risk of CV-morbidity was increased, whereas in postsurgical condition this was not the case (Table 1). It is however unclear, whether the risk for $\mathrm{CV}$ morbidity will increase in patients with postsurgical HypoPT with prolonged observation. Moreover, this large and systematic study demonstrates the safety of conventional therapy (activated vitamin D and calcium supplements) in HypoPT (67).

Further, on NS-HypoPT, a recent cross-sectional study looked at cardiovascular findings in patients with NS-HypoPT compared to Ps-HypoPT (62), addressing the increased CV-risk in patients with the former (68) and seemingly normal risk in the latter disorder (64). Patients with NS-HYpoPT had increased day- and night-time heart rate and blood pressure as compared with Ps-HypoPT. Moreover, as an indicator of CV-risk, PWS was significantly increased in NSHYpoPT (62).

\section{CVD risk in association with biochemical findings in HypoPT}

Lastly, in a recent case-finding study on patients with HypoPT of any cause, Underbjerg et al investigated long-term complications related to biochemical findings (72). Covering about a fifth of the Danish population, data from 431 patients ( $81 \%$ females) with a mean age at the time of diagnosis of 41 years were studied. As expected, most patients (88\%) had postsurgical HypoPT, where the primary surgery was performed for non-malignant disorders. Almost all patients were treated with activated vitamin $\mathrm{D}$ analogues and calcium supplements. The median disease duration was 12.7 years (72). Overall mortality was increased in patients with phosphate levels (and calcium-phosphate products) above the median, compared with levels below the median. Disease duration was associated with an increased overall mortality (as was the risk of CVD). Moreover, more episodes of hypercalcemia was significantly associated with increased mortality and the risk of CVD (72). From a treatment perspective, it is of interest that patients 
treated with higher doses of activated vitamin D was associated with a significantly lower mortality rate compared with doses below the median. Duration of disease was associated with an increased risk of CVD (72) in accordance with the hypothesis referred above for postsurgical HypoPT (67). When looking at calcium levels, low levels were associated with an increased risk of CVD compared with patients with calcium levels in the higher tertiles (72). Again, these data are hypotheses generating only, however important and with perspectives for future management of HypoPT (1). It is though important to emphasize that the generated data describe within group differences only, as there was no control group in the study.

\section{Impact of PTH replacement on CV risk}

Due to the low prevalence of HypoPT large prospective randomized, controlled trials (RCT's) on medical management are not to be expected. Thus for conventional treatment, not such studies have been published. For replacement therapy with the lacking hormone PTH, a few RCT's have been published, but not with CV morbidity as primary outcome parameters (73, 74), neither in the open long-term follow-up studies for up to five years (75). What can be learned so far from these studies is that replacement with PTH on a daily basis for up to five years seems safe. However, cautions should be taken due to the low number of patients concluding the studies.

In a sub-study of the Danish RCT giving $100 \mu \mathrm{g}$ rhPTH(1-84) versus placebo for six months, a total of 39 patients volunteered to perform additional 24 hour investigations at the end of the study without un-blinding the treatment groups (76). In this study, BP and heart rate did not differ between active or placebo treatment, before or after the 6-month trial. In the $24 \mathrm{~h}$ extension study, BP did not differ between the groups, but the heart rate was significantly higher in the $\mathrm{rhPTH}(1-84)$ group up to $10 \mathrm{~h}$ after the injection, compared to the placebo group. In agreement, electrocardiograms showed shorter RR-intervals at the same time points (76). Of interest, plasma concentrations of PTH, 1 hour after the injection correlated positively with heart rate, whereas calcium levels did not (76). The study can be criticized for using a high dose of rhPTH(1-84) leading to hypercalcemia in some patients (74). The positive correlation between PTH (and not calcium) levels and heart rate however, indicates a direct chronotropic effect of the rising level of PTH after the injection. In accordance, the surge in heart rate came within the first hour after the injection and faded first beyond the $10 \mathrm{~h}$ point (76).

In conclusion, sparse data on the heart and CV morbidity following replacement with PTH have been published, and replacement seems safe. Still, subtle data on a potential chronotropic effect immediately following the PTH injection, calls for studies on improving the treatment algorithm in a more physiologic manner (1). 


\section{Discussion and Perspective}

Patients with parathyroid disorders do possess symptoms and morbidity related to the cardiovascular system. Via the specific PTH receptors, the hormone acts on the cardiomyocytes leading to direct chronotropic and indirect inotropic effects. In the vasculature, PTH mediates vasodilatation via smooth muscle relaxation $(63,77,78)$. PTH disturbances are followed by changes in calcium levels also having effects on the vasculature and directly coupled to arteriosclerosis (40). As discussed above, it is difficult to separate the pathophysiological effects of the two mediators in the clinic and in clinical research. In the various international recommendations for managements of parathyroid disorders, focus has mostly been on calcium levels, not PTH (28). This still holds and seems reasonable for the individual patient, primarily due to the stable PTH mediated equilibrium hypercalcemia in PHPT, the fluctuations in PTH levels and not the least analytical challenges for PTH measurements (79). Thus, PTH is the metabolic active hormone and calcium seems to be the most reliable marker of disease activity, at least in PHPT.

The potential threshold for calcium levels in PHPT is based on register - and case-control studies indicating a harming effect above a certain calcium level (27). The point is reflected in the recommendations for management PHPT in accordance with most patients with PHPT in the modern clinic, being diagnosed without specific symptoms $(1,28,80)$. Most probably however, symptoms and morbidity mirror a continuum rather than a threshold, and only large scale RCT on management of mild PHPT can reveal whether a true threshold do exist. So far based on the literature, reversibility of symptoms and morbidity has only been found in more severe PHPT and not consistently in the various RCT's on mild PHPT (48, 49, 81-84). More data are awaited from long-term follow-up data from the Scandinavian SIPH study.

When it comes to the PTH resistant syndromes as in Ps-HypoPT, it is of major interest that the condition seems not to lead to an increase in CVD or CV mortality $(62,64)$. As the resistance to PTH in Ps-HypoPT not necessarily is total in all tissues (15), further studies on the CV system would be of interest to further delineate the effects of PTH and PTH action of relevance for cardiac and CV effects. Moreover, as Ps-HypoPT in many ways is treated like HypoPT, it also underscores the long-term safety of treatment with vitamin D analogues and calcium supplements. There is however different schools in management of HypoPT concerning dosing of vitamin analogues and calcium supplements (70). In Europe, patients are mostly treated with higher doses of activated vitamin D and low (if any) amounts of calcium supplements (18), 
whereas the overseas tradition favors higher amounts of calcium supplements (70). The above mentioned data showing a lower CV-risk in HopoPT patients treated with the higher vitamin D doses compared to the lower raised the hypothesis that a more active attitude in standard treatment of HypoPT in relation to vitamin D analogues might be beneficial from a CV perspective (72).

There is a need for a paradigm shift in management of HypoPT towards a more physiological treatment, with hormonal substitution correcting the two-hormone deficiency state (1). The approved treatment with PTH replacement should be developed further towards a more physiologic algorithm, potential implicating multiple dosing or even pump treatment with PTH itself or (long-acting) PTH analogous (85-87).

\section{Conclusion}

PTH is a metabolic active hormone primarily regulating calcium and phosphate homeostasis in a very tight and short term-manner. The hormone also have more tardive actions directly and indirectly via the two specific G - protein coupled receptors. Endocrine disorders with alterations in PTH secretion or action do have major impact on the cardiovascular system of importance for morbidity and mortality, wherefore management of these specific diseases should be optimized currently, as new data become available, however also avoiding overtreating asymptomatic patients and be aware of potential side effects. 


\section{References}

1. Bollerslev J, Schalin-Jantti C, Rejnmark L, Siggelkow H, Morreau H, Thakker RV, et al. MANAGEMENT OF ENDOCRINE DISEASE: Unmet therapeutic, educational and scientific needs in parathyroid disorders. Eur J Endocrinol. 2019.

2. Fraser WD. Hyperparathyroidism. Lancet. 2009;374(9684):145-58.

3. Bushinsky DA, Monk RD. Electrolyte quintet: Calcium. Lancet. 1998;352(9124):306-11.

4. Bollerslev J, Pretorius $M$, Heck A. Parathyroid hormone independent hypercalcemia in adults. Best practice \& research Clinical endocrinology \& metabolism. 2018;32(5):621-38.

5. Parfitt AM. Large calcium fluxes that are not related to remodeling exist. Bone. 2003;33(3):269.

6. Abou-Samra AB, Juppner H, Force T, Freeman MW, Kong XF, Schipani E, et al. Expression cloning of a common receptor for parathyroid hormone and parathyroid hormone-related peptide from rat osteoblast-like cells: a single receptor stimulates intracellular accumulation of both cAMP and inositol trisphosphates and increases intracellular free calcium. Proc Natl Acad Sci U S A. 1992;89(7):2732-6.

7. Uhlen M, Fagerberg L, Hallstrom BM, Lindskog C, Oksvold P, Mardinoglu A, et al. Proteomics. Tissue-based map of the human proteome. Science (New York, NY). 2015;347(6220):1260419.

8. Usdin TB, Gruber C, Bonner TI. Identification and functional expression of a receptor selectively recognizing parathyroid hormone, the PTH2 receptor. J Biol Chem. 1995;270(26):15455-8.

9. Leifsson BG, Ahren B. Serum calcium and survival in a large health screening program. J Clin Endocrinol Metab. 1996;81(6):2149-53.

10. Lind L, Skarfors E, Berglund L, Lithell H, Ljunghall S. Serum calcium: a new, independent, prospective risk factor for myocardial infarction in middle-aged men followed for 18 years. J Clin Epidemiol. 1997;50(8):967-73.

11. Jorde R, Sundsfjord J, Fitzgerald P, Bonaa KH. Serum calcium and cardiovascular risk factors and diseases: the Tromso study. Hypertension. 1999;34(3):484-90.

12. Hagstrom E, Hellman P, Larsson TE, Ingelsson E, Berglund L, Sundstrom J, et al. Plasma parathyroid hormone and the risk of cardiovascular mortality in the community. Circulation. 2009;119(21):2765-71.

13. Pilz S, Tomaschitz A, Drechsler C, Ritz E, Boehm BO, Grammer TB, et al. Parathyroid hormone level is associated with mortality and cardiovascular events in patients undergoing coronary angiography. Eur Heart J. 2010;31(13):1591-8.

14. Polyzos SA, Duntas L, Bollerslev J. The intriguing connections of leptin to hyperparathyroidism. Endocrine. 2017;57(3):376-87.

15. Mantovani G, Bastepe M, Monk D, de Sanctis L, Thiele S, Usardi A, et al. Diagnosis and management of pseudohypoparathyroidism and related disorders: first international Consensus Statement. Nat Rev Endocrinol. 2018;14(8):476-500.

16. Lundgren E, Rastad J, Thrufjell E, Akerstrom G, Ljunghall S. Population-based screening for primary hyperparathyroidism with serum calcium and parathyroid hormone values in menopausal women. Surgery. 1997;121(3):287-94.

17. Siilin $\mathrm{H}$, Rastad J, Ljunggren $\mathrm{O}$, Lundgren E. Disturbances of calcium homeostasis consistent with mild primary hyperparathyroidism in premenopausal women and associated morbidity. J Clin Endocrinol Metab. 2008;93(1):47-53.

18. Bollerslev J, Rejnmark L, Marcocci C, Shoback DM, Sitges-Serra A, van Biesen W, et al. European Society of Endocrinology Clinical Guideline: Treatment of chronic hypoparathyroidism in adults. Eur J Endocrinol. 2015;173(2):G1-G20. 
19. Elvius M, Lagrelius A, Nygren A, Alveryd A, Christensson TA, Nordenstrom J. Seventeen year follow-up study of bone mass in patients with mild asymptomatic hyperparathyroidism some of whom were operated on. Eur J Surg. 1995;161(12):863-9.

20. Lundstam K, Heck A, Godang K, Mollerup C, Baranowski M, Pernow Y, et al. Effect of Surgery Versus Observation: Skeletal 5-Year Outcomes in a Randomized Trial of Patients With Primary HPT (the SIPH Study). J Bone Miner Res. 2017.

21. Rubin MR, Bilezikian JP, McMahon DJ, Jacobs T, Shane E, Siris E, et al. The natural history of primary hyperparathyroidism with or without parathyroid surgery after 15 years. J Clin Endocrinol Metab. 2008;93(9):3462-70.

22. Bollerslev J. The important players in primary hyperparathyroidism. Clin Endocrinol (Oxf). 2013;79(6):774-5.

23. Yu N, Leese GP, Donnan PT. What predicts adverse outcomes in untreated primary hyperparathyroidism? The Parathyroid Epidemiology and Audit Research Study (PEARS). Clin Endocrinol (Oxf). 2013;79(1):27-34.

24. Rubin MR, Maurer MS, McMahon DJ, Bilezikian JP, Silverberg SJ. Arterial stiffness in mild primary hyperparathyroidism. J Clin Endocrinol Metab. 2005;90(6):3326-30.

25. Persson A, Bollerslev J, Rosen T, Mollerup CL, Franco C, Isaksen GA, et al. Effect of surgery on cardiac structure and function in mild primary hyperparathyroidism. Clin Endocrinol (Oxf). 2011;74(2):174-80.

26. Bollerslev J, Marcocci C, Sosa M, Nordenstrom J, Bouillon R, Mosekilde L. Current evidence for recommendation of surgery, medical treatment and vitamin $D$ repletion in mild primary hyperparathyroidism. Eur J Endocrinol. 2011;165(6):851-64.

27. Vestergaard P, Mollerup CL, Frokjaer VG, Christiansen P, Blichert-Toft M, Mosekilde L. Cardiovascular events before and after surgery for primary hyperparathyroidism. World journal of surgery. 2003;27(2):216-22.

28. Bilezikian JP, Brandi ML, Eastell R, Silverberg SJ, Udelsman R, Marcocci C, et al. Guidelines for the management of asymptomatic primary hyperparathyroidism: summary statement from the Fourth International Workshop. J Clin Endocrinol Metab. 2014;99(10):3561-9.

29. Ong GS, Walsh JP, Stuckey BG, Brown SJ, Rossi E, Ng JL, et al. The importance of measuring ionized calcium in characterizing calcium status and diagnosing primary hyperparathyroidism. J Clin Endocrinol Metab. 2012;97(9):3138-45.

30. Palmer M, Adami HO, Bergstrom R, Akerstrom G, Ljunghall S. Mortality after surgery for primary hyperparathyroidism: a follow-up of 441 patients operated on from 1956 to 1979. Surgery. 1987;102(1):1-7.

31. Hedback G, Tisell LE, Bengtsson BA, Hedman I, Oden A. Premature death in patients operated on for primary hyperparathyroidism. World journal of surgery. 1990;14(6):829-35; discussion 36.

32. Ogard CG, Engholm G, Almdal TP, Vestergaard H. Increased mortality in patients hospitalized with primary hyperparathyroidism during the period 1977-1993 in Denmark. World journal of surgery. 2004;28(1):108-11.

33. Soreide JA, van Heerden JA, Grant CS, Yau Lo C, Schleck C, Ilstrup DM. Survival after surgical treatment for primary hyperparathyroidism. Surgery. 1997;122(6):1117-23.

34. Wermers RA, Khosla S, Atkinson EJ, Grant CS, Hodgson SF, O'Fallon WM, et al. Survival after the diagnosis of hyperparathyroidism: a population-based study. Am J Med. 1998;104(2):11522.

35. Levy D, Garrison RJ, Savage DD, Kannel WB, Castelli WP. Prognostic implications of echocardiographically determined left ventricular mass in the Framingham Heart Study. $\mathrm{N}$ Engl J Med. 1990;322(22):1561-6.

36. Nilsson IL, Aberg J, Rastad J, Lind L. Left ventricular systolic and diastolic function and exercise testing in primary hyperparathyroidism-effects of parathyroidectomy. Surgery. 2000;128(6):895-902. 
37. Nappi S, Saha H, Virtanen V, Limnell V, Sand J, Salmi J, et al. Left ventricular structure and function in primary hyperparathyroidism before and after parathyroidectomy. Cardiology. 2000;93(4):229-33.

38. Walker MD, Fleischer JB, Di Tullio MR, Homma S, Rundek T, Stein EM, et al. Cardiac structure and diastolic function in mild primary hyperparathyroidism. J Clin Endocrinol Metab. 2010;95(5):2172-9.

39. McMahon DJ, Carrelli A, Palmeri N, Zhang C, DiTullio M, Silverberg SJ, et al. Effect of Parathyroidectomy Upon Left Ventricular Mass in Primary Hyperparathyroidism: A MetaAnalysis. J Clin Endocrinol Metab. 2015;100(12):4399-407.

40. Pepe J, Cipriani C, Sonato C, Raimo O, Biamonte F, Minisola S. Cardiovascular manifestations of primary hyperparathyroidism: a narrative review. Eur J Endocrinol. 2017;177(6):R297R308.

41. Kalla A, Krishnamoorthy P, Gopalakrishnan A, Garg J, Patel NC, Figueredo VM. Primary hyperparathyroidism predicts hypertension: Results from the National Inpatient Sample. Int J Cardiol. 2017;227:335-7.

42. Demirtas D, Sumbul HE, Demirtas AO, Icen YK, Gulumsek E, Koca H, et al. Morning blood pressure surge increases in patients with hypertensive primary hyperparathyroidism and is independently associated with serum calcium level. Clin Exp Hypertens. 2020;42(1):86-92.

43. Bolland MJ, Grey AB, Gamble GD, Reid IR. Association between primary hyperparathyroidism and increased body weight: a meta-analysis. J Clin Endocrinol Metab. 2005;90(3):1525-30.

44. Rydberg E, Birgander M, Bondeson AG, Bondeson L, Willenheimer R. Effect of successful parathyroidectomy on 24-hour ambulatory blood pressure in patients with primary hyperparathyroidism. Int J Cardiol. 2010;142(1):15-21.

45. Nilsson IL, Aberg J, Rastad J, Lind L. Maintained normalization of cardiovascular dysfunction 5 years after parathyroidectomy in primary hyperparathyroidism. Surgery. 2005;137(6):632-8.

46. Graff-Baker AN, Bridges LT, Chen Q, Faries MB, Said M. Parathyroidectomy for Patients With Primary Hyperparathyroidism and Associations With Hypertension. JAMA surgery. 2019.

47. Ejlsmark-Svensson H, Rolighed L, Rejnmark L. Effect of Parathyroidectomy on Cardiovascular Risk Factors in Primary Hyperparathyroidism: A Randomized Clinical Trial. J Clin Endocrinol Metab. 2019;104(8):3223-32.

48. Lundstam K, Heck A, Mollerup C, Godang K, Baranowski M, Pernow Y, et al. Effects of parathyroidectomy versus observation on the development of vertebral fractures in mild primary hyperparathyroidism. J Clin Endocrinol Metab. 2015;100(4):1359-67.

49. Bollerslev J, Jansson S, Mollerup CL, Nordenstrom J, Lundgren E, Torring O, et al. Medical observation, compared with parathyroidectomy, for asymptomatic primary hyperparathyroidism: a prospective, randomized trial. J Clin Endocrinol Metab. 2007;92(5):1687-92.

50. Lenzini L, Prisco S, Vanderriele PE, Lerco S, Torresan F, Maiolino G, et al. PTH Modulation by Aldosterone and Angiotensin II is Blunted in Hyperaldosteronism and Rescued by Adrenalectomy. J Clin Endocrinol Metab. 2019;104(9):3726-34.

51. Gomez-Ramirez J, Gomez-Valdazo A, Luengo P, Porrero B, Osorio I, Rivas S. Comparative prospective study on the presentation of normocalcemic primary hyperparathyroidism. Is it more aggressive than the hypercalcemic form? Am J Surg. 2020;219(1):150-3.

52. Cusano NE, Cipriani C, Bilezikian JP. Management of normocalcemic primary hyperparathyroidism. Best practice \& research Clinical endocrinology \& metabolism. 2018;32(6):837-45.

53. Hanon EA, Sturgeon CM, Lamb EJ. Sampling and storage conditions influencing the measurement of parathyroid hormone in blood samples: a systematic review. Clin Chem Lab Med. 2013;51(10):1925-41.

54. Bertocchio JP, Tafflet M, Koumakis E, Maruani G, Vargas-Poussou R, Silve C, et al. Pro-FHH: A Risk Equation to Facilitate the Diagnosis of Parathyroid-Related Hypercalcemia. J Clin Endocrinol Metab. 2018;103(7):2534-42. 
55. Beysel S, Caliskan M, Kizilgul M, Apaydin M, Kan S, Ozbek M, et al. Parathyroidectomy improves cardiovascular risk factors in normocalcemic and hypercalcemic primary hyperparathyroidism. BMC Cardiovasc Disord. 2019;19(1):106.

56. Cansu GB, Yilmaz N, Ozdem S, Balci MK, Suleymanlar G, Arici C, et al. Parathyroidectomy in asymptomatic primary hyperparathyroidism reduces carotid intima-media thickness and arterial stiffness. Clin Endocrinol (Oxf). 2016;84(1):39-47.

57. Schillaci G, Pucci G, Pirro M, Monacelli M, Scarponi AM, Manfredelli MR, et al. Large-artery stiffness: a reversible marker of cardiovascular risk in primary hyperparathyroidism. Atherosclerosis. 2011;218(1):96-101.

58. Rosa J, Raska I, Jr., Wichterle D, Petrak O, Strauch B, Somloova Z, et al. Pulse wave velocity in primary hyperparathyroidism and effect of surgical therapy. Hypertens Res. 2011;34(3):296300.

59. Godang K, Lundstam K, Mollerup C, Fougner F, Pernow Y, Nordenstrom J, et al. The effect of surgery on fat mass, lipid and glucose metabolism in mild primary hyperparathyroidism. Endocr Connect. 2018;7(8):941-8.

60. Brickman AS, Stern N, Sowers JR. Hypertension in pseudohypoparathyroidism type I. Am J Med. 1988;85(6):785-92.

61. Germain-Lee EL, Groman J, Crane JL, Jan de Beur SM, Levine MA. Growth hormone deficiency in pseudohypoparathyroidism type 1a: another manifestation of multihormone resistance. J Clin Endocrinol Metab. 2003;88(9):4059-69.

62. Underbjerg L, Sikjaer T, Rejnmark L. Cardiovascular findings in patients with nonsurgical hypoparathyroidism and pseudohypoparathyroidism: A cohort study. Clin Endocrinol (Oxf). 2019;90(4):592-600.

63. Ogino K, Burkhoff D, Bilezikian JP. The hemodynamic basis for the cardiac effects of parathyroid hormone (PTH) and PTH-related protein. Endocrinology. 1995;136(7):3024-30.

64. Underbjerg L, Sikjaer T, Mosekilde L, Rejnmark L. Pseudohypoparathyroidism - epidemiology, mortality and risk of complications. Clin Endocrinol (Oxf). 2016;84(6):904-11.

65. Astor MC, Lovas K, Debowska A, Eriksen EF, Evang JA, Fossum C, et al. Epidemiology and Health-Related Quality of Life in Hypoparathyroidism in Norway. J Clin Endocrinol Metab. 2016;101(8):3045-53.

66. Clarke BL, Brown EM, Collins MT, Juppner H, Lakatos $P$, Levine MA, et al. Epidemiology and Diagnosis of Hypoparathyroidism. J Clin Endocrinol Metab. 2016;101(6):2284-99.

67. Underbjerg L, Sikjaer T, Mosekilde L, Rejnmark L. Cardiovascular and renal complications to postsurgical hypoparathyroidism: a Danish nationwide controlled historic follow-up study. J Bone Miner Res. 2013;28(11):2277-85.

68. Underbjerg L, Sikjaer T, Mosekilde L, Rejnmark L. The Epidemiology of Nonsurgical Hypoparathyroidism in Denmark: A Nationwide Case Finding Study. J Bone Miner Res. 2015;30(9):1738-44.

69. Bansal B, Bansal M, Bajpai P, Garewal HK. Hypocalcemic cardiomyopathy-different mechanisms in adult and pediatric cases. J Clin Endocrinol Metab. 2014;99(8):2627-32.

70. Khan AA, Koch C, Van Uum SHM, Baillargeon JP, Bollerslev J, Brandi ML, et al. Standards of Care for Hypoparathyroidism in Adults. Eur J Endocrinol. 2018.

71. Brandi ML, Bilezikian JP, Shoback D, Bouillon R, Clarke BL, Thakker RV, et al. Management of Hypoparathyroidism: Summary Statement and Guidelines. J Clin Endocrinol Metab. 2016;101(6):2273-83.

72. Underbjerg L, Sikjaer T, Rejnmark L. Long-Term Complications in Patients With Hypoparathyroidism Evaluated by Biochemical Findings: A Case-Control Study. J Bone Miner Res. 2018;33(5):822-31.

73. Mannstadt M, Clarke BL, Vokes T, Brandi ML, Ranganath L, Fraser WD, et al. Efficacy and safety of recombinant human parathyroid hormone (1-84) in hypoparathyroidism (REPLACE): a double-blind, placebo-controlled, randomised, phase 3 study. Lancet Diabetes Endocrinol. 2013;1(4):275-83. 
74. Sikjaer T, Rejnmark L, Rolighed L, Heickendorff L, Mosekilde L. The effect of adding PTH(1-84) to conventional treatment of hypoparathyroidism: a randomized, placebo-controlled study. J Bone Miner Res. 2011;26(10):2358-70.

75. Mannstadt M, Clarke BL, Bilezikian JP, Bone H, Denham D, Levine MA, et al. Safety and Efficacy of 5 Years of Treatment With Recombinant Human Parathyroid Hormone in Adults With Hypoparathyroidism. J Clin Endocrinol Metab. 2019;104(11):5136-47.

76. Sikjaer T, Amstrup AK, Rolighed L, Kjaer SG, Mosekilde L, Rejnmark L. PTH(1-84) replacement therapy in hypoparathyroidism: a randomized controlled trial on pharmacokinetic and dynamic effects after 6 months of treatment. J Bone Miner Res. 2013;28(10):2232-43.

77. Schluter KD, Piper HM. Cardiovascular actions of parathyroid hormone and parathyroid hormone-related peptide. Cardiovasc Res. 1998;37(1):34-41.

78. Brown SJ, Ruppe MD, Tabatabai LS. The Parathyroid Gland and Heart Disease. Methodist Debakey Cardiovasc J. 2017;13(2):49-54.

79. Smit MA, van Kinschot CMJ, van der Linden J, van Noord C, Kos S. Clinical Guidelines and PTH Measurement: Does Assay Generation Matter? Endocr Rev. 2019;40(6):1468-80.

80. Khan AA, Hanley DA, Rizzoli R, Bollerslev J, Young JE, Rejnmark L, et al. Primary hyperparathyroidism: review and recommendations on evaluation, diagnosis, and management. A Canadian and international consensus. Osteoporos Int. 2017;28(1):1-19.

81. Ambrogini E, Cetani F, Cianferotti L, Vignali E, Banti C, Viccica G, et al. Surgery or surveillance for mild asymptomatic primary hyperparathyroidism: a prospective, randomized clinical trial. J Clin Endocrinol Metab. 2007;92(8):3114-21.

82. Bollerslev J, Rosen T, Mollerup CL, Nordenstrom J, Baranowski M, Franco C, et al. Effect of surgery on cardiovascular risk factors in mild primary hyperparathyroidism. J Clin Endocrinol Metab. 2009;94(7):2255-61.

83. Lundstam K, Heck A, Godang K, Mollerup C, Baranowski M, Pernow Y, et al. Effect of Surgery Versus Observation: Skeletal 5-Year Outcomes in a Randomized Trial of Patients With Primary HPT (the SIPH Study). J Bone Miner Res. 2017;32(9):1907-14.

84. Rao DS, Phillips ER, Divine GW, Talpos GB. Randomized controlled clinical trial of surgery versus no surgery in patients with mild asymptomatic primary hyperparathyroidism. J Clin Endocrinol Metab. 2004;89(11):5415-22.

85. Winer KK. Advances in the treatment of hypoparathyroidism with PTH 1-34. Bone. 2019;120:535-41.

86. Krishnan V, Ma YL, Chen CZ, Thorne N, Bullock H, Tawa G, et al. Repurposing a novel parathyroid hormone analogue to treat hypoparathyroidism. Br J Pharmacol. 2018;175(2):262-71.

87. Holten-Andersen L, Pihl S, Rasmussen CE, Zettler J, Maitro G, Baron J, et al. Design and Preclinical Development of TransCon PTH, an Investigational Sustained-Release PTH Replacement Therapy for Hypoparathyroidism. J Bone Miner Res. 2019;34(11):2075-86. 


\section{Tables}

Table 1. Risk of cardiovascular diseases in patients with postsurgical- and non-surgicalhypoparathyroidism. Hazard ratio (95\% confidence interval). Significant risk estimates are high-lighted in bold. Adapted from reference $(67,68)$

\section{Mortality}

Any cardiovascular disease

Myocardial infarction, acute

Ischemic heart disease

Stroke

Arrhythmia

Cardiac arrest

\begin{tabular}{cc} 
Post-surgical & Non-surgical \\
\hline $0.98(0.76-1.28)$ & $1.25(0.90-1.73)$ \\
$0.89(0.73-1.09)$ & $\mathbf{1 . 9 1}(\mathbf{1 . 2 9}-\mathbf{2 . 8 9})$ \\
$0.77(0.44-1.34)$ & $1.29(0.61-2.72)$ \\
$1.09(0.83-1.45)$ & $\mathbf{2 . 0 1 ( 1 . 3 1 - 3 . 0 9 )}$ \\
$1.09(0.73-1.64)$ & $1.84(0.95-3.54)$ \\
$1.11(0.79-1.57)$ & $1.78(0.96-3.30)$ \\
$0.68(0.15-3.23)$ & $2.84(0.40-20.13)$ \\
\hline
\end{tabular}




\section{Figures}

Figure 1

Immunohistochemical staining of PTHR1 and PTHR2 in human tissue. A) PTHR1 is detected in the cortex of kidney, labeling show weak to moderate positivity in renal tubules with a polyclonal antibody (HPA075879, http://www.proteinatlas.org/). The staining is relatively weak, but selective to kidney and no positivity is observed in other tissue types. The pictures otherwise unpublished, courtesy Dr. Evelina Sjöstedt, the Human Protein Atlas project, Sweden. B) PTHR2 is detected in pancreas as well as endothelial cells throughout the whole body, including renal glomeruli. Images show example of brain, heart muscle, and submucosal layer of intestine where blood vessels are positively labeled using a polycloncal antibody (HPA010655). PTHR2 positivity is visualized using two different dilution factors of the original antibody concentration of $0.08 \mathrm{mg} / \mathrm{ml}$ (conc. $1-0.04 \mathrm{ug} / \mathrm{ml}$, conc. $2-0.16 \mathrm{ug} / \mathrm{ml}$ ) indicating a less strong but still specific labeling of exocrine pancreas, renal glomeruli as well as blood vessels throughout the body.

A
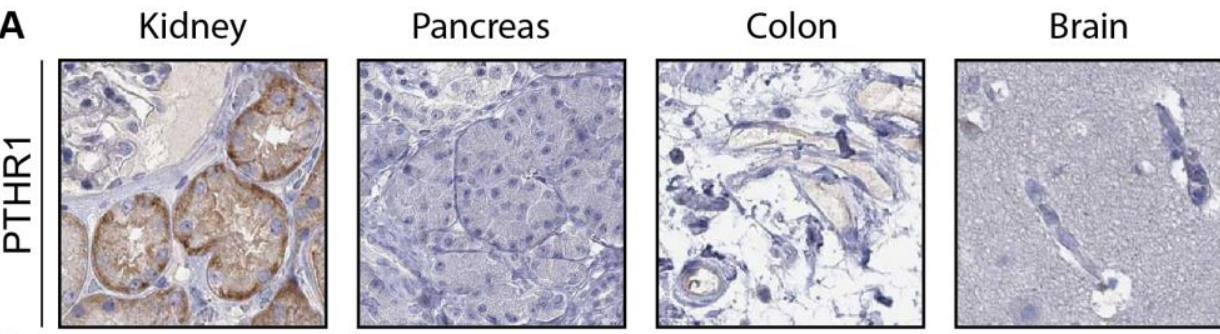

Heart muscle
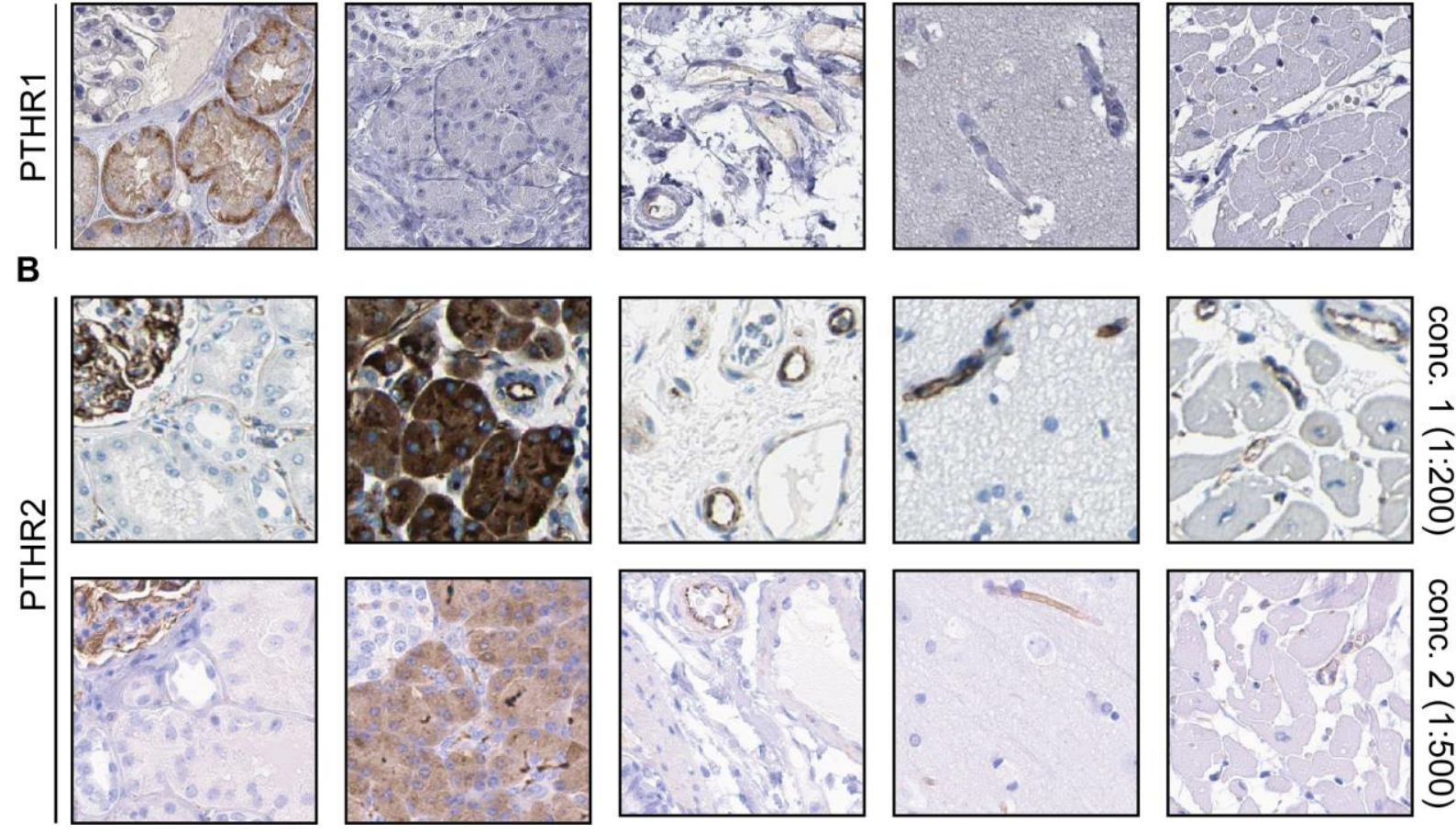